\title{
In Vitro activity of a Novel Benzoquinolizine Antibiotic, Levonadifloxacin (WCK 771) against Blood Stream Gram-Positive Isolates from a Tertiary Care Hospital
} \author{
Jaishid Ahdal ${ }^{3}$ Rishi Jain ${ }^{3}$ \\ 1Department of Microbiology, S.L. Raheja Hospital, Mumbai, \\ Maharashtra, India \\ 2Department of Critical Care Medicine, S.L. Raheja Hospital, \\ Mumbai, Maharashtra, India \\ ${ }^{3}$ Department of Medical Affairs, Wockhardt Ltd., Mumbai, \\ Maharashtra, India \\ ${ }^{4}$ Drug Discovery Research, Wockhardt Research Center, \\ Aurangabad, Maharashtra, India
}

Dhruv Mamtora ${ }^{1}$ Sanjith Saseedharan ${ }^{2}$ Ritika Rampal ${ }^{3} \quad$ Prashant Joshi $^{4} \quad$ Pallavi Bhalekar $^{1}$

J Lab Physicians:2020;12:230-232

\begin{abstract}
Address for correspondence Dhruv Mamtora, MD, Department of Microbiology, S.L. Raheja Hospital, Raheja Rugnalaya Marg, Mahim West, Mahim, Mumbai, Maharashtra 400016, India (e-mail: dhruv_mamtora@yahoo.com).
\end{abstract}

\begin{abstract}
Keywords

- blood stream

infections

- methicillin-resistant

S. aureus

- levonadifloxacin

- quinolone-resistant

S. aureus
\end{abstract}

Background Blood stream infections (BSIs) due to Gram-positive pathogens such as methicillin-resistant Staphylococcus aureus (MRSA) are associated with high mortality ranging from 10 to $60 \%$. The current anti-MRSA agents have limitations with regards to safety and tolerability profile which limits their prolonged usage. Levonadifloxacin and its oral prodrug alalevonadifloxacin, a novel benzoquinolizine antibiotic, have recently been approved for acute bacterial skin and skin structure infections including diabetic foot infections and concurrent bacteremia in India.

Methods The present study assessed the potency of levonadifloxacin, a novel benzoquinolizine antibiotic, against Gram-positive blood stream clinical isolates $(n=31)$ collected from January to June 2019 at a tertiary care hospital in Mumbai, India. The susceptibility of isolates to antibacterial agents was defined following the Clinical and Laboratory Standard Institute interpretive criteria (M100 E29).

Results High prevalence of MRSA (62.5\%), quinolone-resistant Staphylococcus aureus (QRSA) (87.5\%), and methicillin-resistant coagulase-negative staphylococci (MR-CoNS) (82.35\%) were observed among bacteremic isolates. Levonadifloxacin demonstrated potent activity against MRSA, QRSA, and MR-CoNS strains with significantly lower minimum inhibitory concentration $\mathrm{MIC}_{50 / 90}$ values of $0.5 / 1 \mathrm{mg} / \mathrm{L}$ as compared with levofloxacin $(8 / 32 \mathrm{mg} / \mathrm{L})$ and moxifloxacin $(2 / 8 \mathrm{mg} / \mathrm{L})$.

Conclusion Potent bactericidal activity coupled with low MICs support usage of levonadifloxacin for the management of BSIs caused by multidrug resistant Gram-positive bacteria.

\section{Introduction}

With more than 90 years from the discovery of penicillin, bacterial infections are still the leading cause of morbidity and mortality worldwide including India. Blood stream infections (BSIs) caused by Gram-positive pathogens may originate from diverse sources such as pneumonia, urinary tract infection, acute bacterial skin and skin structure
DOI https://doi.org/ 10.1055/s-0040-1720944 ISSN 0974-2727. (c) 2020. The Indian Association of Laboratory Physicians.

This is an open access article published by Thieme under the terms of the Creative Commons Attribution-NonDerivative-NonCommercial-License, permitting copying and reproduction so long as the original work is given appropriate credit. Contents may not be used for commercial purposes, or adapted, remixed, transformed or built upon. (https://creativecommons.org/licenses/by-nc-nd/4.0/)

Thieme Medical and Scientific Publishers Pvt. Ltd., A-12, 2nd Floor, Sector 2, Noida-201301 UP, India 
infection (ABSSSI), and catheter-associated infections. In the event of suboptimal treatment, BSI may result in further complications such as infective endocarditis and sepsis. Mortality rates ranging from 40 to $60 \%$ and prolonged length of intensive care unit stay have been observed with BSI. ${ }^{1}$ The Indian Network for Surveillance of Antimicrobial Resistance has reported the methicillin-resistant Staphylococcus aureus (MRSA) bacteremia rates to be $48 \% .^{2}$ Further, infection with MRSA is found to be an independent predictor of mortality in $\mathrm{BSI} .{ }^{3,4}$

Management of MRSA in India, especially in BSI is quite challenging. This can be attributed to ineffectiveness of the current anti-MRSA agents (e.g., vancomycin, linezolid, daptomycin). Both vancomycin and linezolid lack rapid cidal action which restricts their clinical utility in the treatment of BSI. Despite the availability of vancomycin, which is a standard therapy for BSI management, the mortality rates were reported to be $>30 \%{ }^{5}$ Reports have listed vancomycin minimum inhibitory concentration (MIC) creep, variable tissue penetration, and nephrotoxicity at high serum concentrations, as major challenges in its clinical use. ${ }^{6}$ Linezolid is also not indicated to be used in staphylococcal bacteremia due to its bacteriostatic mode of action. Moreover, prolonged usage of linezolid is not approved because of adverse effects such as thrombocytopenia, optic, and peripheral neuropathy. ${ }^{7}$ On the other hand, daptomycin, even though cidal, is ineffective in pneumonia (a major source for gram-positive BSI) as it is inactivated by pulmonary surfactant ${ }^{8}$ and is also shown to have cross-resistance with vancomycin in heteroresistant vancomycin-intermediate Staphylococcus aureus (hVISA) strains. ${ }^{9}$ Hence, there is a need for a new drug having rapid bactericidal action, low probability of resistance development, and lower risk of side effects, to achieve maximum microbiological and clinical cure in BSI.

Levonadifloxacin-IV (WCK 771) and its oral L-alanine prodrug, alalevonadifloxacin (WCK 2349), are novel broad spectrum antibiotics belonging to benzoquinolizine subclass of fluoroquinolone, approved in India for the indication of ABSSSI with concurrent bacteremia and diabetic foot infections caused by susceptible Gram-positive pathogens. The distinctive novel structural feature of a nonbasic hydroxypiperidine side chain at C-8 position of tricyclic benzoquinolizine core confers a well differentiated mode of action to levonadifloxacin as compared with other quinolones. It primarily targets deoxyribonucleic acid gyrase which allows it to demonstrate high potency even against commonly prevalent quinolone-resistant Staphylococcus aureus (QRSA) that harbors multiple mutations in topoisomerase
IV..$^{10}$ Levonadifloxacin not only exhibit bactericidal effect on biofilm-embedded QRSA and MRSA but also inhibits NorA efflux pump which is a major cause of quinolone resistance development. Moreover, levonadifloxacin has an effective cidal action against high density cultures. ${ }^{11}$ In the present study, we tested in vitro activity of levonadifloxacin against BSI gram-positive clinical isolates.

\section{Methods}

The study was conducted between January and June 2019 at S.L. Raheja Hospital, Mumbai, Maharashtra, India, and has been approved by the institutional ethics committee (CLS/2017/SLRH). The study isolates $(n=31)$ were collected as nonduplicate samples from individual patients suspected with BSI potentially caused by Gram-positive pathogens. Organisms responsible for BSI were later identified by biochemical and molecular methods. The identified organisms included S. epidermidis $(n=17)$, S. aureus $(n=8)$, and Enterococci spp. $(n=6)$.

\section{Susceptibility Testing}

The MICs of levonadifloxacin and other comparator antibiotics were determined by the reference agar dilution method as described by the Clinical and Laboratory Standard Institute (CLSI). ${ }^{12}$ Susceptibility of levonadifloxacin was defined on the basis of susceptibility breakpoint of $2 \mathrm{mg} / \mathrm{L}$ for Gram-positive pathogens. The susceptibility of other antibacterial agents was defined following interpretive criteria presented in the CLSI document M100 edition 29. ${ }^{13}$ S. aureus ATCC 29213 was included in each run as quality control strain.

\section{Results and Discussion}

Out of 31 isolates, $54.8 \%$ were caused by S. epidermidis followed by S. aureus (25.8\%) and Enterococci (19.3\%). Among these clinical isolates, high prevalence of MRSA (62.5\%), QRSA (87.5\%), and methicillin-resistant coagulase-negative staphylococci (MR-CoNS, 82.35\%) was observed. Levonadifloxacin showed best-in-class activity against collected Staphylococci isolates $(n=25)$ which included MRSA $(n=8)$, QRSA $(n=8)$, and MR-CoNS $(n=17)$. Levonadifloxacin demonstrated significantly lower $\mathrm{MIC}_{50 / 90}$ values of $0.5 / 1 \mathrm{mg} / \mathrm{L}$ as compared with levofloxacin $(8 / 32 \mathrm{mg} / \mathrm{L})$ and moxifloxacin $(2 / 8 \mathrm{mg} / \mathrm{L})$ against these isolates (-Table $\mathbf{1})$. - Table 2 illustrates MIC values of levonadifloxacin in comparison to vancomycin, linezolid, and teicoplanin against Staphylococci isolates.

Table 1 Cumulative distribution of MICs of levonadifloxacin in comparison to levofloxacin and moxifloxacin

\begin{tabular}{|c|c|c|c|c|c|c|c|c|c|c|c|c|}
\hline \multirow[t]{2}{*}{ Antibacterial } & \multicolumn{12}{|c|}{ \% cumulative inhibition of Staphylococci spp. at respective MIC (mg/L) } \\
\hline & 0.03 & 0.06 & 0.12 & 0.25 & 0.5 & 1 & 2 & 4 & 8 & 16 & 32 & $>32$ \\
\hline $\begin{array}{l}\text { Levonadifloxacin } \\
\text { (WCK 771) }\end{array}$ & $3(12.0)$ & $3(24.0)$ & $0(24.0)$ & $0(24.0)$ & $8(56.0)$ & $9(92.0)$ & $2(100)$ & $0(100)$ & $0(100)$ & $0(100)$ & $0(100)$ & $0(100)$ \\
\hline Levofloxacin & 0 & 0 & $1(4.0)$ & $3(16.0)$ & $1(20.0)$ & $1(24.0)$ & $0(24.0)$ & $5(44.0)$ & $9(80.0)$ & $2(88.0)$ & $2(96.0)$ & $1(100)$ \\
\hline Moxifloxacin & 0 & $3(12.0)$ & $0(12.0)$ & $3(24.0)$ & $0(24.0)$ & $4(40.0)$ & $9(76.0)$ & $3(88.0)$ & $3(100)$ & $0(100)$ & $0(100)$ & $0(100)$ \\
\hline
\end{tabular}

Abbreviation: MIC, minimum inhibitory concentration. 
Table 2 Susceptibility profiling of levonadifloxacin and comparators against Staphylococci spp.

\begin{tabular}{|l|l|l|l|l|l|l|}
\hline Antibacterial agent & Range & MIC $_{50}$ & MIC $_{90}$ & S & I & R \\
\hline Levonadifloxacin (WCK 771) & $0.03-2$ & 0.5 & 1 & $100^{\text {a }}$ & - & - \\
\hline Moxifloxacin & $0.06-8$ & 2 & 2 & 24 & 16 & 60 \\
\hline Levofloxacin & $0.12->32$ & 8 & 8 & 24 & 0 & 76 \\
\hline Vancomycin & $0.5-2$ & 1 & 1 & 100 & 0 & 0 \\
\hline Linezolid & $0.5-8$ & 1 & 1 & 96 & - & 4 \\
\hline Teicoplanin & $0.5-16$ & 2 & 2 & 96 & 4 & 0 \\
\hline Co-trimoxazole & $0.06->32$ & 0.5 & 0.5 & 80 & - & 20 \\
\hline
\end{tabular}

Abbreviation: MIC, minimum inhibitory concentration.

a Susceptibility of levonadifloxacin was defined on the bases of susceptibility break point of $2 \mathrm{mg} / \mathrm{L}$.

Enterococci are known to display high level of resistance to most of the antibiotic classes. In such challenging scenarios, levonadifloxacin MICs were found to be lower $(2-16 \mathrm{mg} / \mathrm{L})$ than moxifloxacin $(2-32 \mathrm{mg} / \mathrm{L})$ and levofloxacin $(>32 \mathrm{mg} / \mathrm{L})$. The potency of comparator anti-MRSA drugs vancomycin (MIC range $0.5-16 \mathrm{mg} / \mathrm{L}$ ) and linezolid (MIC range $1-8 \mathrm{mg} / \mathrm{L}$ ) were in expected range.

In the past, several susceptibility testing studies undertaken with levonadifloxacin demonstrated consistent $\mathrm{MIC}_{90}$ of 0.5 to $1 \mathrm{mg} / \mathrm{L}$ against variety of Gram-positive pathogens. For instance, its potent activity has been reported against 1,438 MRSA isolates with MIC $_{90}$ of $1 \mathrm{mg} / \mathrm{L}$ in a global surveillance study. ${ }^{14}$ Similarly, against 308 QRSA isolates collected in a recent multicenter study, conducted in India, $\mathrm{MIC}_{90}$ of $1 \mathrm{mg} / \mathrm{L}$ was reported in comparison to the $\mathrm{MIC}_{90}$ of $16 \mathrm{mg} / \mathrm{L}$ for levofloxacin. ${ }^{15}$ Therefore, the present study result are in agreement with the previous studies which substantiate the consistent activity profile of levonadifloxacin. In summary, potent bactericidal activity of levonadifloxacin coupled with promising activity observed in the present study support clinical use of levonadifloxacin for difficult-to-treat Gram-positive BSIs.

\section{Authors' Contribution}

R.R., P.J., and P.B.: Data generation and analysis, drafting of the manuscript, and critical revision. D.M., S.S., J.A., and R.J.: Study concept and design, study supervision, data interpretation, critical revision, and final approval of the manuscript.

\section{Conflict of Interest}

D.M., S.S., and P.B. have nothing to declare. R.R., P.J., J.A., and R.J. are employees of Wockhardt Ltd.

\section{References}

1 Bassetti M, Righi E, Carnelutti A. Bloodstream infections in the intensive care unit. Virulence 2016;7(3):267-279

2 Indian Network for Surveillance of Antimicrobial Resistance (INSAR) group, India. Methicillin resistant Staphylococcus aureus (MRSA) in India: prevalence \& susceptibility pattern. Indian J Med Res 2013;137(2):363-369

3 Prowle JR, Echeverri JE, Ligabo EV, et al. Acquired bloodstream infection in the intensive care unit: incidence and attributable mortality. Crit Care 2011;15(2):R100
4 Bassetti M, Trecarichi EM, Mesini A, et al. Risk factors and mortality of healthcare-associated and community-acquired Staphylococcus aureus bacteraemia. Clin Microbiol Infect 2012;18(9):862-869

5 Pastagia M, Kleinman LC, Lacerda de la Cruz EG, Jenkins SG. Predicting risk for death from MRSA bacteremia. Emerg Infect Dis 2012;18(7):1072-1080

6 Nguyen HM, Graber CJ. Limitations of antibiotic options for invasive infections caused by methicillin-resistant Staphylococcus aureus: is combination therapy the answer. ? J Antimicrob Chemother 2010;65(1):24-36

7 Narita M, Tsuji BT, Yu VL. Linezolid-associated peripheral and optic neuropathy, lactic acidosis, and serotonin syndrome. Pharmacotherapy 2007;27(8):1189-1197

8 Potashman MH, Formella DN, Hamed K, Mohr JF. Comment on: efficacy and safety of daptomycin for the treatment of infectious disease: a meta-analysis based on randomized controlled trials. J Antimicrob Chemother 2015;70(4):1274-1275

9 Cui L, Tominaga E, Neoh HM, Hiramatsu K. Correlation between reduced daptomycin susceptibility and vancomycin resistance in vancomycin-intermediate Staphylococcus aureus. Antimicrob Agents Chemother 2006;50(3):1079-1082

10 Bhagwat SS, Mundkur LA, Gupte SV, Patel MV, Khorakiwala HF. The anti-methicillin-resistant Staphylococcus aureus quinolone WCK 771 has potent activity against sequentially selected mutants, has a narrow mutant selection window against quinolone-resistant Staphylococcus aureus, and preferentially targets DNA gyrase. Antimicrob Agents Chemother 2006;50(11):3568-3579

11 Bhagwat SS, Nandanwar M, Kansagara A, et al. Levonadifloxacin, a novel broad-spectrum anti-MRSA benzoquinolizine quinolone agent: review of current evidence. Drug Des Devel Ther 2019;13:4351-4365

12 Clinical and Laboratory Standard Institute (CLSI), 11 th ed 2018

13 Clinical and Laboratory Standard Institute (CLSI), 29th ed 2019

14 Flamm RK, Farrell DJ, Sader HS, et al. In Vitro Activity of WCK 771, a Benzoquinolizine Fluoroquinolone (Levonadifloxacin) when Tested Against Contemporary Gram-Positive and -Negative Bacteria from a Global Surveillance Program. Poster: Sunday 456, MICROBE, June 16-20, 2016. Boston, MA, USA

15 Appalaraju B, Baveja S, Baliga S, et al. In vitro activity of a novel antibacterial agent, levonadifloxacin, against clinical isolates collected in a prospective, multicentre surveillance study in India during 2016-18. J Antimicrob Chemother 2020;75(3):600-608 